Journal of Patient-Centered

7-27-2020

\title{
Solutions to Address Frequent Hospital Attendance
}

Glenda Sundberg

Follow this and additional works at: https://aah.org/jpcrr

Part of the Clinical and Medical Social Work Commons, Community Health and Preventive Medicine Commons, Family Practice Nursing Commons, Interprofessional Education Commons, Medical Humanities Commons, Other Medicine and Health Sciences Commons, Primary Care Commons, and the Public Health and Community Nursing Commons

\section{Recommended Citation}

Sundberg G. Solutions to address frequent hospital attendance. J Patient Cent Res Rev. 2020;7:222-6. doi: 10.17294/2330-0698.1786

Published quarterly by Midwest-based health system Advocate Aurora Health and indexed in PubMed Central, the Journal of Patient-Centered Research and Reviews (JPCRR) is an open access, peer-reviewed medical journal focused on disseminating scholarly works devoted to improving patient-centered care practices, health outcomes, and the patient experience. 


\title{
EDITORIAL
}

\section{Solutions to Address Frequent Hospital Attendance}

\author{
Glenda Sundberg, FNP-CS \\ Department of Family Medicine, Aurora UW Medical Group, Advocate Aurora Health, Milwaukee, WI
}

$\mathrm{I}$ $\mathrm{t}$ was a pleasure to read the review article by Shukla and colleagues - published in this issue of the Journal of Patient-Centered Research and Reviews (p. 255) - which provides a more uniform definition of, and an explanation of factors correlating to, frequent attendance (FA). ${ }^{1}$ As the authors reported, there is indeed limited literature on how to best serve those meeting the criteria of FA in the community and within our health systems. ${ }^{2}$ Compassion and caring for others with integrity and respect must be at the core of what we do as health care providers. There is no one more deserving of compassionate care than those most vulnerable, including patients with high health system utilization and the families and friends who care for them at home.

Speaking from a primary care perspective, all providers have a panel of patients and each includes a certain few who have high needs and meet the criteria of FA. ${ }^{2,3}$ In managing these medically complex, socially at-risk patients, there is a point when one realizes that all their needs cannot be met effectively, as some of the needs expand beyond the capabilities of the clinic office environment. This experience can trigger emotions of hopelessness and frustration that, over time, can lead to provider apathy and burnout. ${ }^{4}$

One way to approach reaching the needs of complex patient care in a more sustainable manner is to move toward more collaborative models. ${ }^{5}$ In addressing the needs of complex patients, such as in the case of

Correspondence: Glenda Sundberg, FNP-CS, AUWMG Family Practice Center, 2801 W. Kinnickinnic River Parkway, \#250, Milwaukee, WI 53215 (glenda.sundberg@aah.org) patients with FA, the aim should be to create models that are neither patient- nor clinician-centered but that are more compassionate and honest. ${ }^{6}$ It has been suggested that models that embrace the Quadruple Aim of health care by improving the patient experience, population health concerns, health cost, and caregiver well-being may be of great benefit. ${ }^{7-9}$ How one accomplishes this within the infrastructures of the health care industry as it exists today is indeed the challenge. The intention of this editorial is to provide practical solutions to this dilemma.

The solution to reaching and providing proper care for those with FA is multifactorial and perhaps as complex as the patients we are trying to help. One approach to achieving successful care models focuses on four dimensions: service setting, care model attributes, care delivery features, and organizational culture. ${ }^{10} \mathrm{~A}$ care program's effectiveness and efficiency increase when it targets the people most likely to benefit from specific interventions. ${ }^{11}$

Regarding service setting, there are a variety of sustainable evidence-based models that exist across health care venues in the community, long-term care, and acute care settings. Some focus on managing transitions of care from hospital to home, with success evidenced by improved patient outcomes and a decrease in health care costs. Better Outcomes by Optimizing Safe Transitions (BOOST) and Transitions to Care are two specific models of this nature, among others. ${ }^{12,13}$

Two examples of care models that have shown success with a focus on interdisciplinary, personcentered primary care are the Geriatric Resources for Assessment and Care of Elders (GRACE) program and the Program of All-Inclusive Care for the Elderly (PACE). ${ }^{14-23}$ These models were implemented because 
traditional primary care models were not designed to fully support the complex needs of elderly adults. Within these models, patients with identified needs are placed into patient groups and given more handson care by interdisciplinary teams. Teams meet face to face to work on improving comprehensive case management, care coordination, and creating individualized care plans. ${ }^{14-23}$

The GRACE model includes an in-home assessment and care management completed by nurse practitioners and community social workers (NP/ SW). ${ }^{14-16}$ Assessment includes a geriatric assessment, functional assessment, and environmental scan. $\mathrm{NP} / \mathrm{SW}$ meet weekly with a geriatrician and other team members, including a pharmacist, mental health professional, and community-based services representative. Results of this model reveal high patient and physician satisfaction. There was greater improvements related to health in general and geriatric conditions, including falls and depression. The program also resulted in lower overall resource use, lower cost, and less emergency room and hospital visits. ${ }^{14-16}$ Models like these tend to complement the care that is already being given to the patient from primary care as opposed to competing with it. ${ }^{18}$

PACE is a Medicare- and Medicaid-covered program that helps people meet their health care needs in the community instead of admission to a nursing home or other care facility. ${ }^{19-23}$ A team of health professionals works with families to ensure that patients receive coordinated care at home. ${ }^{19}$ PACE participants report they are healthier, happier, and more independent than their counterparts in other care settings. ${ }^{20}$ They also live longer than enrollees in other home- and communitybased waiver programs. ${ }^{20}$ PACE reduces the need for costly long-term nursing home care, prevents and/or significantly reduces preventable hospitalizations, and produces Medicare savings. ${ }^{21-24}$ While the GRACE and PACE models are designed to manage the vulnerable elderly population, these types of models have the potential to be applied to other at-risk or vulnerable populations, such as patients with FA.

Organizational culture is important. The implementation processes of these models encounter difficulties in gaining momentum or producing lasting change in health systems. ${ }^{21,24,25}$ Those working to deploy BOOST observed that the hospitals with the greatest success in implementing the program were the ones whose senior leadership prioritized the program. ${ }^{24}$ One example would be administrators who gave frontline staff the time and resources needed to do BOOST-related work by freeing them of some of their other responsibilities. One BOOST team member stated, "Nobody in the hospital these days is looking for more to do and people are quite resistant to change." ${ }^{24}$ Organizations that invested toward successful implementation, for example, by paying a mentor for a year to implement the program's interventions, had better overall success. ${ }^{24}$ These issues are critical to consider when creating and implementing future models.

Findings from other past research can help guide the creation of future models. Including community health workers on interdisciplinary teams has shown benefit in improving patient knowledge, medication adherence, and lowering blood pressure and cholesterol levels; this strategy was especially helpful when working with diverse populations. ${ }^{25}$ Freeing up staff to attend team meetings is an ongoing challenge for many interdisciplinary teams. ${ }^{26}$ Although case management programs can be incorporated into traditional primary care, these efforts often fail to engage primary care clinicians, individuals who may know these complex patients best. ${ }^{17}$

Another source suggested that it is imperative to gain a better understanding of who the high-cost patients truly are. Most high-cost patients, it can be argued, are not the "super-utilizers" with frequent admissions, or FA. ${ }^{27}$ The authors asserted that effective interventions to reduce costs require more multifaceted approaches to the patients who trigger them. ${ }^{27}$ Tochukwu et al pointed out that there is a broad range of nonmedical risk factors that play a greater role than previously recognized in early hospital readmission of lowincome seniors (perhaps more than mental health and medical complexity). ${ }^{28}$ There is also evidence of reluctance to dedicate health system financial resources to addressing the social determinants of health. This reluctance is problematic, given that attention to nonmedical issues has the potential for lowering health care costs. ${ }^{29}$ 
In an extensive review of what works well in care model implementation, Kirst et al recommended the following considerations to promote a model's success: creating trusting multidisciplinary team relationships, encouraging provider commitment to and understanding of the model, and having strong leadership that sets clear goals and establishes an organizational culture in support of the program. ${ }^{30}$ Time and flexibility are needed to build infrastructure during the implementation process of models. ${ }^{30}$

Currently, in a fee-for-service health care environment, patients receive expensive, fragmented care that does not fully account for their needs. ${ }^{17,31}$ In this feefor-service reimbursement system, implementing new programs often requires up-front investments, but the financial results, including reduced use of hospitalization and emergency care, contradict the incentives. ${ }^{31}$ New alternative payment models aimed at limiting wasteful spending while improving quality, such as accountable care organizations, may be well suited for medically complex and socially at-risk patients with FA. ${ }^{32}$ Innovators of new models are needed, but they may struggle with making a compelling business case for adoption of the models they are proposing in the current health care climate. ${ }^{17}$

A recent randomized control trial was done to test the Camden Coalition hot-spotting program, a care model for super-utilizers of the health system initiated after hospital discharge. ${ }^{33,34}$ In this particular team model, nurses, social workers, and community health workers conduct visits on enrolled patients to coordinate outpatient care and link them with social services. ${ }^{33}$ One finding was that the team's interventions demonstrated no effect on hospital readmissions, indicating the inability of fractured health care and social service systems to adequately support complex patients, even with the support of experienced teams. ${ }^{34}$ Ultimately, this highlights the importance of advocating for systemwide changes that bridge gaps between health care and social services. This may include implementing medication-assisted treatment for opioid use disorder, addressing housing instability through accessible and affordable housing programs, and establishing partnerships between medicine and law professionals to tackle the legal barriers faced by some patients.
In my opinion, just because the Camden model did not affect readmission rates does not mean that comprehensive hot spotting of patients with FA should be abandoned. Allowing space for clinicians to get out in the community, connect with resources, and get into patients' homes is a helpful approach that can yield positive results and improve health outcomes.

One such model is working well for our primary care residency program in Milwaukee, Wisconsin..$^{35-37}$ Currently in its fourth year of implementation, this primary care-focused, interdisciplinary model is designed to help our most at-risk patients and has been successfully tailored to meet the unique needs of 3 clinic sites. The interdisciplinary teams comprise physicians (both faculty and residents), nurse practitioners, clinical pharmacists, social workers, and nurses. Within the model, teams work collaboratively throughout the year on a predetermined number of patients referred to the program; 2-hour in-person team meetings are conducted 3 times per year to review all cases. Inclusion in the program does not require FA (though some patients we follow would certainly meet that criterion), but rather direct referrals are taken from the primary care clinicians we work with every day. When referring into the program, clinicians send information as to why they are referring and what the concerns are. Initial evaluation of an accepted patient includes a full assessment of not only social determinants of health, but also social structures, language, cultural, and other health disparities. Polypharmacy, medication management, and noncompliance issues are evaluated, as well as safety in the home and other insurance or financial concerns. Each patient enrolled in the program receives at least one home visit. ${ }^{35}$

Our experience with the implementation process of these models across our sites is that interdisciplinary teams that collaborate well can effectively address the unique complex needs of each patient. Over the past 4 years, our model has demonstrated 1) a high level of patient satisfaction, 2) a great level of acceptance by providers, 3) an overwhelmingly large number of patients being referred for the program, and 4) cost savings; thereby accomplishing health care's Quadruple Aim. ${ }^{35-37}$ 
Development of potential solutions to patient frequent attendance will indeed benefit from a uniform definition of FA. What should follow for these patients is a different approach to better understand the unique circumstances that are triggering their FA. Innovative models that can aid in bridging system gaps, achieving better health outcomes, and improving the wellbeing of both patients and providers are needed and should be explored. It takes a culture of openness and creativity, coupled with passion from strong teams, to effectively implement such multifaceted models and powerfully motivate care providers to enact change across health systems and communities.

These are the types of care approaches worth creating and supporting. They reflect compassionate humanity, which is at the core of what we all do. It matters.

\section{Acknowledgments}

The author thanks Dennis Baumgardner, MD, of Aurora UW Medical Group for his editing contributions to this work and ongoing support, and also Veronica Daniels, a University of Wisconsin School of Medicine and Public Health (Madison, WI) and TRIUMPH program medical student who helped with the literature review and editing.

\section{References}

1. Shukla DM, Faber EB, Sick B. Defining and characterizing frequent attenders: systematic literature review and recommendations. J Patient Cent Res Rev. 2020;7:255-64.

2. Kivelä K, Elo S, Kääriäinen M. Frequent attenders in primary health care: a concept analysis. Int J Nurs Stud. 2018;86:11524. CrossRef

3. Schoen C, Osborn R, Doty MM, Squires D, Peugh J, Applebaum S. A survey of primary care physicians in eleven counties, 2009: perspectives on care, costs, and experiences. Health Aff (Millwood). 2009;28:w1171-83. CrossRef

4. Kung A, Cheung T, Knox M, et al. Capacity to address social needs affects primary care clinician burnout. Ann Fam Med. 2019;17:487-94. CrossRef

5. Cacchione PZ. Innovative care models across settings: providing nursing care to older adults. Geriatr Nurs. 2020;41:16-20. CrossRef

6. McNally PJ, Charlton R, Ratnapalan M, Dambha-Miller H. Empathy, transference and compassion. J $R$ Soc Med. 2019;112:420-3. CrossRef

7. Coidakis-Barss C, Patchell A. Close the Divide. Interprofessional education and a collaborative culture will help healthcare organizations achieve the Quadruple Aim. Published 2019 Apr 1. https://www.td.org/magazines/tdmagazine/close-the-divide. TD Magazine. 2019;73(4):32.

8. Hsieh D. Achieving the Quadruple Aim: treating patients as people by screening for and addressing the social determinants of health. Ann Emerg Med. 2019;74:S19-24. CrossRef
9. Wagner E, LeRoy L, Schaefer J, et al. How do innovative primary care practices achieve the Quadruple Aim? J Ambul Care Manag. 2018;41:288-97. CrossRef

10. Figueroa J. Approach for achieving effective care for highneed patients. N Engl J Med. 2018;178:845-6. CrossRef

11. Blumenthal D, Chernof, B, Fulmer T, Lumpkin J, Selberg J. Caring for high-need, high-cost patients - an urgent priority. N Engl J Med. 2016;375:909-11. CrossRef

12. Chan B, Edwards ST, Devoe $M$, et al. The SUMMIT ambulatory-ICU primary care model for medically and socially complex patients in an urban Federally Qualified Health Center: study design and rationale. Addict Sci Clin Pract. 2018;13:27. CrossRef

13. Bellon JE, Bilderback A, Ahuja-Yende NS, et al. University of Pittsburgh Medical Center home transitions multidisciplinary care coordination reduces readmissions for older adults. $J \mathrm{Am}$ Geriatr Soc. 2019;67:156-63. CrossRef

14. Kubat B. The amazing GRACE care team model. Caring for the Ages. 2016;17(5):6-7. CrossRef

15. Counsell SR, Callahan CM, Buttar AB, Clark DO, Frank KI. Geriatric Resources for Assessment and Care of Elders (GRACE): a new model of primary care for low-income seniors. J Am Geriatr Soc. 2006;54:1136-41. CrossRef

16. Ritchie $\mathrm{C}$, Andersen R, Eng J, et al. Implementation of an interdisciplinary, team-based complex care support health care model at an academic medical center: impact on health care utilization and quality of life. PLoS One. 2016;11(2):e0148096. CrossRef

17. Hochman M, Asch SM. Disruptive models in primary care: caring for high-needs, high-cost populations. J Gen Intern Med. 2017;32:392-7. CrossRef

18. Boult C, Wieland GD. Comprehensive primary care for older patients with multiple chronic conditions: "Nobody rushes you through.” JAMA. 2010;304:1936-43. CrossRef

19. Wieland D, Boland R, Baskins J, Kinosian B. Five-year survival in a program of all-inclusive care for elderly compared with alternative institutional and home- and community-based care. J Gerontol A Biol Sci Med Sci. 2010;65:721-6. CrossRef

20. Leavitt MO, Secretary of Health and Human Services. Interim report to Congress. The quality and cost of the Program of All-inclusive Care for the Elderly (PACE). Memorandum issued 2009. https://bluestempace.org/assets/uploads/pace/ media/Leavitt+Interim+Report+to+Congress.pdf. Accessed June 30, 2020.

21. Massachusetts Division of Health Care Finance and Policy, Executive Office of Elder Affairs. PACE evaluation summary (2005). Unpublished work, cited at: https://bluestempace.org/ assets/uploads/pace/media/5+Key+Research+Findings.pdf. Accessed June 30, 2020.

22. Damons J. Program of All-Inclusive Care for the Elderly (PACE) year 2 overview. Nashville, TN: Bureau of TennCare, 2001.

23. Friedman SM, Steinwachs DM, Rathouz PJ, Burton LC, Mukamel DB. Characteristics predicting nursing home admission in the Program of All-Inclusive Care for Elderly people. Gerontologist. 2005;45:157-66. CrossRef

24. Williams MV, Li J, Hansen LO, et al. Project BOOST implementation: lessons learned. South Med J. 2014;107:455-65. $\underline{\text { CrossRef }}$ 
25. Franklin CM, Bernhardt JM, Lopez RP, Long-Middleton ER, Davis S. Interprofessional teamwork and collaboration between community health workers and healthcare teams: an integrative review. Health Serv Res Manag Epidemiol. 2015;2: 2333392815573312. CrossRef

26. Dattalo FM, Nothelle S, Tackett S, et al. Frontline account: targeting hot spotters in an internal medicine residency clinic. J Gen Intern Med. 2014;29:1305-7. CrossRef

27. Lee NS, Whitman N, Vakharia N, Taksler GB, Rothberg MB. High-cost patients: hot-spotters don't explain the half of it. $J$ Gen Intern Med. 2017;32:28-34. CrossRef

28. Iloabuchi TC, Deming M, Tu W, Counsell SR. Risk factors for early hospital readmission in low-income elderly adults. $J \mathrm{Am}$ Geriatr Soc. 2014;62:489-94. CrossRef

29. Stone RI. Investing in the social dimensions of health. Community-based programs can bridge the medical and social worlds to meet older adults' needs. Generations. 2019;(Winter 2019-20 supplement):63-9.

30. Kirst M, Im J, Burns T, et al. What works in implementation of integrated care programs for older adults with complex needs? A realist review. Int J Qual Health Care. 2017;29:612-24. CrossRef

31. Japinga M, Alexander M, McClellan MB. Value-based payment reform for serious illness. Highlighting progress and evidence-based steps that could accelerate the transformation of serious illness care. Generations. 2019;(Winter 2019-20 supplement):35-41.
32. Kaufman BG, Spivack BS, Stearns SC, Song PH, O’Brien EC. Impact of accountable care organizations on utilization, care, and outcomes: a systematic review. Med Care Res Rev. 2019;76:255-90. CrossRef

33. Gawande A. The hot spotters. The New Yorker. 2011 Jan 17. https://www.newyorker.com/magazine/2011/01/24/the-hotspotters. Accessed June 30, 2020.

34. Finkelstein A, Zhou A, Taubman S, Doyle J. Health care hotspotting - a randomized, controlled trial. $N$ Engl $J$ Med. 2020;382:152-62. CrossRef

35. St Clair MC, Sundberg G, Kram JJ. Incorporating home visits in a primary care residency clinic: the patient and physician experience. J Patient Cent Res Rev. 2019;6:203-9. CrossRef

36. Sundberg G, Peters C, de Grandville C, Sorenson N, Patel R, Grube M. Hot spotting medically complex at-risk patients in an urban primary care residency clinic. (abstr.) J Patient Cent Res Rev. 2018;5:319. CrossRef

37. Grube M, Sundberg G, Olsen D, Kram JJ. Hot spotting medically complex at-risk patients: Does it decrease hospital and emergency department admissions? (abstr.) J Patient Cent Res Rev. 2019;6:283-4. CrossRef

(C) 2020 Aurora Health Care, Inc. 